\title{
Design of a Short Reference Non-Coherent Chaos-Based Communication Systems
}

\author{
Georges Kaddoum*, Member, IEEE, Ebrahim Soujeri, Senior Member, IEEE, Yogesh Nijsure, Member, IEEE
}

\begin{abstract}
Data rate and energy efficiency decrement caused by the transmission of reference and data carrier signals in equal portions constitute the major drawback of differential chaos shift keying (DCSK) systems. To overcome this dominant drawback, a short reference DCSK system (SR-DCSK) is proposed. In SR-DCSK, the number of chaotic samples that constitute the reference signal is shortened to $R$ such that it occupies less than half of the bit duration. To build the transmitted data signal, $P$ concatenated replicas of $R$ are used to spread the data. This operation increases data rate and enhances energy efficiency without imposing extra complexity onto the system structure. The receiver uses its knowledge of the integers $R$ and $P$ to recover the data. The proposed system is analytically studied and the enhanced data rate and bit energy saving percentages are computed. Furthermore, theoretical performance for AWGN and multipath fading channels are derived and validated via simulation. In addition, optimising the length of the reference signal $R$ is exposed to detailed discussion and analysis. Finally, the application of the proposed short reference technique to the majority of transmit reference systems such as DCSK, multicarrier DCSK, and quadratic chaos shift keying enhances the overall performance of this class of chaotic modulations and is, therefore, promising.
\end{abstract}

Index Terms-Non-coherent chaos based communication system, SR-DCSK, High data rate, Energy efficiency, Optimization, Performance analysis.

\section{INTRODUCTION}

Coherent communications dominate the state-of-the-art of the wireless communications domain. Such systems require the perfect knowledge of channel state information at the receiver side to perform the demodulation of the received information. Signal pilots that rely on training-oriented channel computation approaches are utilised in this direction. Nonetheless, estimation techniques like this augment the overhead in the signal and the complexity of the receiver and create obstacles to efficient designs. This is why coherent systems are said not to be really implementable in fast-fading situations where the coherence time is truly short.

Furthermore, the transmitter must intermittently send pilot symbols in slow-fading channels, which leads to exhausting available reserves especially in situations where pilot transmissions are excessive.

Added to this is the deterioration caused by flawed and erroneous channel estimations, which constitute another detriment for coherent communications.

G. Kaddoum, E. Soujeri and Y. Nijsure are with University of Québec, ÉTS, LaCIME Laboratory, 1100 Notre-Dame west, H3C 1K3, Montreal, Canada (email: georges.kaddoum@etsmtl.ca). E. Soujeri is also affiliated to the Dept. of Electrical and Electronics Eng., European University of Lefke, North Cyprus.

* This work has been supported by the NSERC discovery grant $435243-$ 2013.
Considering the disadvantages of coherent communications, few of which are discussed above, and taking into consideration the fact that non-coherent detection schemes do neither require the knowledge of any channel state information nor demand any complex synchronisation processes between transmitting and receiving parties, a diverse number of research buddies have actually invested in the field of non-coherent chaos-based communication systems.

Taking into consideration the fact that chaotic signals have plenty of advantages like superb correlation properties, wideband peculiarities and easily-done generation, the use of this category of waveforms for wireless communications is recommended by many researchers [1], [2] and in particular for multiuser spread-spectrum communications [1], [3][7]. Chaos-based modulations provide the features mentioned herein while it does not cross the red lines preserved by spreadspectrum modulations like security of communications [8], jamming resistance and low probability of interception (LPI) [9] as well as mitigation of fading in time varying channels [10].

In the last 15 years, many coherent and non-coherent chaosbased communication systems have been studied and inspected [7], [11]-[17]. Conventional differential chaos shift keying (DCSK) [2], [13], [16]-[18] is one of the most examined systems amid chaos-based communication systems simply because neither chaotic synchronisation nor channel state information is a requirement at the receiver side to generate the required chaotic replica. Interestingly, the only requirement is the symbol or frame rate sampling.

Two equivalent time slots constitute the bit duration in a conventional DCSK system. The reference chaotic signal is placed in the first slot, and the product of the reference signal by the transmitted data is placed in the second slot. As a result, the second slot will either carry the reference signal itself if the transmitted bit is positive, or a negated version of the reference signal if the transmitted bit is negative.

It must be indicated that both DCSK and differential phase shift keying (DPSK) modulations are non-coherent schemes and do not require channel state information at the receiver to recover the transmitted data [10], [13]. But, in comparison to DPSK, the DCSK is more robust to multipath fading environments and is far more suitable for ultra wide band (UWB) applications [7], [10], [13], [16], [19].

Nonetheless, the main drawback of conventional DCSK is due to the repetition of chaos sequences which causes data rate to be slow and half of the bit energy to be wasted.

Starting by the early work of [20] in which a quadratic chaos shift keying QCSK is proposed, which uses the Hilbert 
transform to generate orthogonal bases of chaotic functions in order to allow the transmission of more bits with respect to conventional DCSK while demanding an equal amount of bandwidth and finishing by the multi-resolution M-ary DCSK scheme of [21], there has been a series of serious efforts to outstrip the shortages of DCSK.

In a similar manner, a high efficiency DCSK (HE-DCSK) is proposed in [22], a work in which the modulator recycles each reference slot and two bits of data can be carried in one datamodulated sample sequence. This increases the bandwidth efficiency by twofold, makes the transmitted signal less prone to interception by minimising re-transmissions, but system complexity is increased in comparison to DCSK.

Furthermore, multi-carrier (MC-DCSK) is introduced in [7] and is extended to the multiuser case in [23], [24]. In this architecture, the transmission of chaotic reference sequences is done over a predefined subcarrier frequency while multiple modulated data streams are transmitted over the remaining subcarriers. The MC-DCSK system offers increased data rates and improves energy efficiency.

An improved DCSK (I-DCSK) system is lately proposed in [25] in which the reference signal is added to the data carrier signal after time reversal. This is done via establishing orthogonality between these two signals in order to halve the duration of the transmitted symbol which naturally leads to doubling the spectral efficiency of the I-DCSK system.

Contributions and paper outline: The majority of proposed schemes suffer from high system complexity or low energy efficiency. In this paper we address for the first time the question of reducing the length of the reference signal of the transmit reference non-coherent chaos-based communication systems. The main motivation of our paper is to propose a non-coherent DCSK scheme that has an improved data rate and energy efficiency, and that avoids complex designs and performs without the use of any channel estimator at the receiver. In this new design, the reference signal is shortened such that it consists of $R$ samples instead of $\beta$ samples where $R<\beta$. To construct the transmitted symbol and maintain the same recovered bit energy like in DCSK scheme, $P$ replicas of the reference signal are concatenated such that it has a total length of $\beta=P \cdot R$ and this concatenation is used as a signature waveform to spread the data information symbol. The total bit length in our proposed design would be $T_{b}=R+P R=R+\beta<2 \beta$. Compare this to the conventional DCSK where the transmitted bit length after spreading is $2 \beta$. At the receiver, the reference signal is extracted from the header of each bit frame (by sampling the first $R$ chips out of $R+P R$ chips that constitute the total bit length) then $P$ correlations, each with length $R$, are carried out with the remaining part of the bit duration. The $P$ individual number of correlations are summed up (algebraic addition) and the outcome is compared to an unbiased threshold to recover the transmitted data. In this scenario, the receiver does not need any complex channel estimator, nor does it need to know the content of the reference signal. Shortening the reference signal reduces the bit duration, which increases the data rate and enhances the energy efficiency of the system without sacrificing the BER of the system or increasing its complexity.
Analytical bit error rate expressions over multipath fading and additive white Gaussian noise (AWGN) channels are derived and verified through numerical evaluations. Moreover, an approach to calculate the optimal length of the reference signal $R$ is presented and discussed. Our results show that the length $R$ must be lower than the data carrier length for an enhanced performance. These results will open up the door to new future non-coherent designs taking into account this property.

Since the proposed approach can be applied to the majority of transmit reference non-coherent systems as part of the global solution to enhance this class of chaotic modulation schemes, we extend our approach to cover QCSK and MCDCSK as well. Finally, the performance of the mentioned systems are evaluated and compared with and without the shortened reference method. The contributions of this paper are summarized as follows

1) Proposing a short reference DCSK system design.

2) Evaluating the energy efficiency and the data rate enhancement with respect to conventional DCSK system.

3) Deriving the optimal reference signal length.

4) Computing and analysing the BER performance over multipath fading and AWGN channels.

The remainder of this paper is organized as follows: In section II, the conventional DCSK is briefly presented and the architecture of the SR-DCSK system is explained. Performance analysis of the SR-DCSK scheme is done in section III. An analysis of the reference length and a derivation of its optimal value is put forward in section IV. Simulation results and discussions are presented in section $\mathrm{V}$ and concluding remarks are presented in section VI.

\section{NON-COHERENT CHAOS-BASED COMMUNICATION SYSTEMS}

\section{A. DCSK Communication System}

Each bit $b_{i}=\{-1,+1\}$ in the DCSK modulator [1] is represented by two sets of chaotic signal samples placed in two consecutive time slots of equal lengths. The first time slot is allocated to the reference signal, and the second slot is allocated to the data carrier. The content of the data carrier slot is the product (multiplication) of the reference signal by the transmitted bit, i.e. the reference signal spreads the transmitted bit. In simpler terms, the content of the second slot will either be the reference signal or an inverted version of the reference signal depending on the transmitted bit.

Similar to the spreading gain in CDMA communications, the spreading factor in DCSK systems is defined as the number of chaotic samples used to spread each transmitted bit and is presented by $2 \beta$, where $\beta$ is an integer. Furthermore, we define $T_{D C S K}=2 T_{b}=2 \beta T_{c}$ to be the DCSK frame time interval for each transmitted bit.

The discrete form of the baseband signal sequence of the $i^{\text {th }}$ bit time interval at the output of the $k^{\text {th }}$ transmitter $e_{k, i}$, may be expressed as

$$
e_{k, i}=\left\{\begin{array}{ll}
x_{k, i} & \text { for }(0<k \leq \beta) \\
b_{i} x_{k-\beta, i} & \text { for }(\beta<k \leq 2 \beta)
\end{array},\right.
$$


where $x_{k, i}$ is the chaotic sequence used as the reference signal and $x_{k-\beta, i}$ is its delayed version. In addition $E_{b, i}=2 \beta E\left[x_{k, i}^{2}\right]$ represents the transmitted bit energy of DCSK modulation. In order to demodulate the transmitted bits, the received signal $r_{k}$ is correlated with its delayed version $r_{k+\beta}$ and summed over the bit duration $T_{b}$ where $T_{b}=\beta T_{c}$ and $T_{c}$ is the chip time. The received bits are then estimated by comparing the correlator output to a zero threshold. In the design of such a system, the recovered bit energy after demodulation is equal to $E r_{b, i}=\beta E\left[x_{k, i}^{2}\right]$. Hence, in this system half of the frame bit time interval is dedicated to the non-information-bearing reference signal which dissipates half the energy of each bit $E_{b, i}$. This fact decreases data rate and energy efficiency in the DCSK system.

\section{B. SR-DCSK System Architecture}

As illustrated in Fig.1 (a), for each bit $b_{i}$, a chaotic signal that consists of $R$ samples (chips) is generated and used as a reference signal. The reference signal is replicated and concatenated $P$ times in order to form the data carrier sequence with a length of $\beta=P \cdot R$ samples and maintains the same recovered bit energy $E r_{b, i}$ after demodulation, like DCSK scheme. The information bit $b_{i}$ is transmitted after being multiplied (spread) by the concatenation of the reference sequence of length $\beta$. Hence, the bit frame of SR-DCSK system is formed with a reference sequence of $R$ samples and a data carrier signal with $\beta$ samples where $\beta=P \cdot R$, for a total of $R+\beta=R+P \cdot R$ samples. Moreover, we can clearly see from Fig.1 (b) and (c) that the SR-DCSK frame saves more time and energy compared to DCSK frames. So, instead of generating $\beta$ samples as done in DCSK scheme, just $R$ samples are generated and used as shortened reference signal. This operation aims at reducing the DCSK frame time to increase data rate and enhance energy efficiency. Next sections will analyse the length of the reference signal $R$ with respect to energy and data rate enhancements, and system performance. In our system, the baseband discrete transmitted signal may be expressed as

$$
e_{k, i}= \begin{cases}x_{k, i} & \text { for }(0 \leq k<R) \\ b_{i} x_{k-R, i} & \text { for }(R \leq k \leq(1+P) R) \\ \text { and } x_{k-R, i} \equiv x_{0, i} \bmod (R)\end{cases}
$$

As shown in Fig.1 (d), in order to demodulate the transmitted bits, the received signal $r_{k, i}$ must first be delayed with a delay of $R$ in order to allow the extraction of the reference signal which exists in the first $R$ samples of the SR-DCSK frame. The reference signal is then partially correlated over $P$ consecutive samples of the frame, each with length $R$. Finally, the $P$ independent correlation values are then summed up (algebraic addition) and an estimation of the transmitted bit is obtained by comparing the outcome to a zero threshold.

With this frame design, the corresponding frame duration becomes $T_{S R-D C S K}=(R+\beta) T_{c}$. The SR-DCSK system increases the data rate compared to DCSK by a factor of
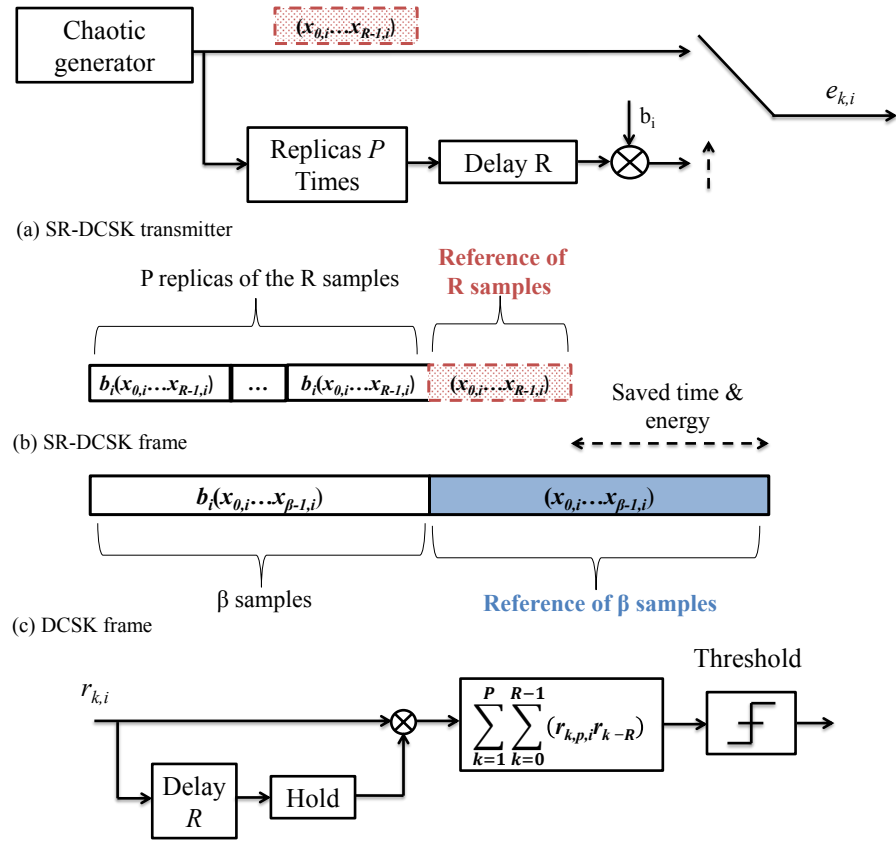

(d) SR-DCSK receiver

Fig. 1: (a) Block diagram of the general structure of the SR-DCSK transmitter; (b) SR-DCSK frame (c) DCSK frame and (d) SR-DCSK receiver.

$$
E_{D}=\frac{R_{S R-D C S K}-R_{D C S K}}{R_{D C S K}} \times 100 \%,
$$

where $E_{D}$ is the enhancement percentage in data rate of SRDCSK compared to DCSK system, $R_{S R-D C S K}=\frac{1}{(\beta+R)}$ and $R_{D C S K}=\frac{1}{(2 \beta)}$ represent the data rate of SR-DCSK and DCSK systems, respectively. Another way to represent $E_{D}$ would be to say

$$
E_{D}=2 \beta\left(\frac{1}{(\beta+R)}-\frac{1}{(2 \beta)}\right) \times 100 \%,
$$

which would simplify to

$$
E_{D}=\frac{(\beta-R)}{(\beta+R)} \times 100 \%,
$$

In a similar fashion, the ratio of energy saving in SR-DCSK compared to conventional DCSK would be

$$
S_{E}=\frac{E_{b, D C S K}-E_{b, S R-D C S K}}{E_{b, D C S K}} \times 100 \%,
$$

where $S_{E}$ represents the percentage of the saved energy compared to DCSK system, $E_{b, S R-D C S K}=T_{c}(\beta+R) E\left[x_{k}^{2}\right]$ and $E_{b, D C S K}=2 T_{c} \beta E\left[x_{k}^{2}\right]$ represent the total transmitted bit energy of of SR-DCSK and DCSK systems, respectively. The above equation may be expressed in simpler terms as

$$
S_{E}=\frac{(\beta+R)}{2 \beta} \times 100 \% .
$$

Fig. 2 shows the amount of data rate enhancement and energy saving of SR-DCSK compared to DCSK for different 
values of $R$ when $\beta=100$. Based on equation (5), we observe that for a reference length equal to zero, i.e. $R=0$, the system reaches its maximal data rate speed which represents $100 \%$ of enhancement compared to DCSK system. In other words, the SR-DCSK system doubles its data rate compared to DCSK system. Vis a vis, when the reference signal and the data carrier have equal lengths, i.e. $R=\beta$, the SR-DCSK reduces to DCSK and the enhancement is $0 \%$. Similarly, the energy savings presented by $E_{S}$ varies from $50 \%$ to $100 \%$ when the length $R$ varies from 0 to $\beta$. In the upcoming sections we analyse the impact of the reference length, i.e. the value of $R$, on the system performance.

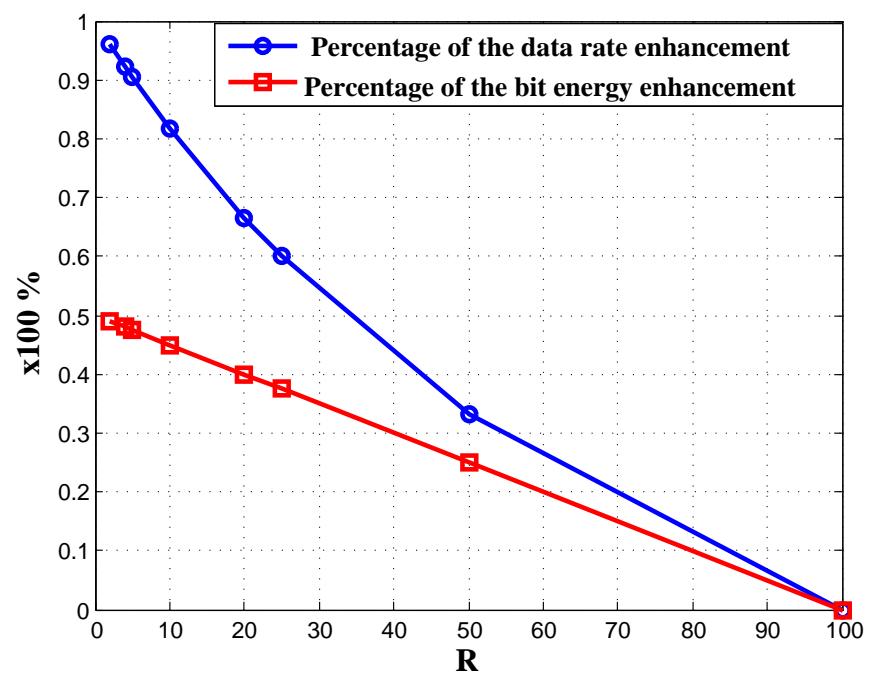

Fig. 2: Data rate and bit energy enhancement percentages of SRDCSK compared to DCSK for a spreading factor $\beta=100$.

In this paper a multipath fading channel with $L$ independent paths is considered. It should be noted that the channel coefficients follow Rayleigh distribution. Therefore, the probability density function of the channel coefficient $\alpha$ in this case can be given as

$$
f(\alpha \mid \sigma)=\frac{\alpha}{\sigma^{2}} e^{-\frac{\alpha^{2}}{2 \sigma^{2}}},
$$

where $\sigma>0$ is the scale parameter of the distribution representing the root mean square value of the received voltage signal before envelope detection.

The baseband discreet received signal could be be written as

$$
r_{k, i}=\sum_{l=1}^{L} \alpha_{l, i} e_{k-\tau_{l}, i}+n_{k},
$$

where $\alpha_{l, i}$ and $\tau_{l}$ are the channel gain and the delay of the $l^{\text {th }}$ path, $L$ is the number of paths and $n_{k}$ is additive white Gaussian noise with zero mean and variance $N_{0} / 2$. At the receiver side, the receiver recovers the reference signal from the head of each frame, then performs $P$ partial correlations over each block of $R$ samples and the obtained $P$ values are summed and compared to the threshold to recover the data. In our analysis, we assume that the largest delay $\tau_{L}$ is much shorter than the reference duration such that

$$
0<\tau_{L}<<R .
$$

In this case, the inter-symbol interference (ISI) is negligible [7], [10]. The limit of this assumption is discussed in the simulation section. Moreover, we also assume that the channel is slowly fading such that channel coefficients are constant during a given SR-DCSK symbol. Finally, since the transmitted data signal is periodic with period length equal to $R$, the output of the partial correlation becomes

$$
\begin{gathered}
O_{i, p}=T_{c} \sum_{k=0}^{R-1}\left(\sum_{l=1}^{L} \alpha_{l, i} x_{k-\tau_{l}, i} b_{i}+n_{p, k+R}\right) \\
\left(\sum_{l=1}^{L} \alpha_{l, i} x_{k-\tau_{l}, i}+n_{k}\right) .
\end{gathered}
$$

where $O_{i, p}$ is the output value of the $p^{\text {th }}$ partial correlation, $n_{p, k+R}$ is $k^{\text {th }}$ AWGN noise added to the $k$ sample of the $p^{\text {th }}$ data signal slot, and $n_{k}$ is the AWGN noise added to the reference signal. It is important to note that the noise signal $n_{k}$ remains constant over all $P$ partial correlations which have an effect on the statistical properties of the decision variable.

For large reference length $R$, the following approximated expression is used:

$$
\sum_{k=1}^{R} x_{k-\tau_{l}} x_{k-\tau_{m}} \approx 0, l \neq m .
$$

Hence, using the approximation in equation (12), the variable $O_{i, p}$ may be further simplified and expanded as

$$
\begin{gathered}
O_{i, p}=T_{c} \sum_{k=0}^{R-1}(\underbrace{\sum_{l=1}^{L} \alpha_{l, i}^{2} x_{k-\tau_{l}, i}^{2} b_{i}}_{U}+\underbrace{n_{k} n_{p, k+R}}_{N_{1}} \\
+\underbrace{n_{k}\left(\sum_{l=1}^{L} \alpha_{l, i} x_{k-\tau_{l}, i} b_{i}\right)}_{N_{2}}+\underbrace{n_{p, k+R}\left(\sum_{l=1}^{L} \alpha_{l, i} x_{k-\tau_{l}, i}\right)}_{N_{3}}),
\end{gathered}
$$

where $U$ represents the useful signal, $N_{1}, N_{2}$ and $N_{3}$ designate interference emerging from Gaussian noise. For mathematical simplification the chip duration is assumed to be $T_{c}=1$.

The decision variable of the SR-DCSK system can be written as

$$
D_{i}=\sum_{p=1}^{P} O_{i, p} .
$$

Since the data carrier exhibits a periodic shape and each slot $p$ is correlated by the same reference having $R$ chaotic and noise samples, we can observe that the noise component $n_{k}$, and the chaotic codes $x_{k, i}$ and $x_{k-\tau, i}$ keep the same values, independent from index of the data carrier slot $p$. The decision variable may, therefore, be expanded as

$$
D_{i}=P \sum_{k=0}^{R-1} U+\underbrace{P \sum_{k=0}^{R-1}\left(N_{2}\right)}_{T I_{1}}+\underbrace{\sum_{p=1}^{P} \sum_{k=0}^{R-1}\left(N_{3}+N_{1}\right)}_{T I_{2}} .
$$




\section{Performance Analysis of SR-DCSK System}

In this section, the performance of SR-DCSK scheme is evaluated and the BER expressions under AWGN and multipath fading channels are analytically derived. To this end, the mean and the variance expressions of the decision variable $D_{i}$ are determined. The signal components $U, N_{1}, N_{2}$ and $N_{3}$ in equation (13) are independent because the channel coefficients are independent of each other, of the chaotic sequences and of the Gaussian noise. In addition, the chaotic sequence is independent of the Gaussian noise as well [1].

In this work, the second-order Chebyshev polynomial function $(\mathrm{CPF})$ is employed to generate chaotic sequences due to its easiness and good performance [26], i.e.

$$
x_{k+1}=1-2 x_{k}^{2} .
$$

The variance of the normalized chaotic map with zero mean is equal to one, i.e. $\mathrm{V}[x]=\mathrm{E}\left[x^{2}\right]=1$, where $\mathrm{V}[\cdot]$ denotes the variance operator. Therefore, for the $i^{\text {th }}$ bit, the instantaneous mean of the decision variable $D_{i}$ is the mean of the useful signal part given by

$$
E\left[D_{i}\right]=R P b_{1} \sum_{l=1}^{L} \alpha_{l, i}^{2} E\left[x_{k, i}^{2}\right],
$$

where $R P E\left[x_{k, i}^{2}\right]$ represents the recovered bit energy from $P$ correlations. This recovered energy is equal to the recovered on in DCSK system. As we can observe, equation (17) is reduced to the mean of the useful signal component $U$ because all other components described in equation (13) have zero mean. On the other hand, by replacing the term $E\left[x_{k, i}^{2}\right]$ by the bit energy expression of SR-DCSK where $E_{b}=(R+\beta) E\left[x_{k, i}^{2}\right]$, the expression in equation (17) will be

$$
E\left[D_{i}\right]=\frac{R P}{(R+\beta)} b_{1} \sum_{l=1}^{L} \alpha_{l, i}^{2} E_{b} .
$$

Since all the components in the decision variable are independent, the conditional variance is the sum of the variances of all of the interference components.

The variances of $N_{1}, N_{2}$, and $N_{3}$ can be computed based on the expression developed in equation (13). Hence, based on the independence property of random variables, the variance of the first total interference component $T I_{1}$ of the decision variable given in equation (15) can be expanded as

$$
V\left[T I_{1}\right]=P^{2} V\left[\sum_{k=0}^{R-1} N_{2}\right] .
$$

Based on equation (13), the variance of the first term in equation (19) is

$$
V\left[N_{2}\right]=E\left[x_{k-\tau_{l}, i}^{2}\right] \frac{N_{0}}{2} \sum_{l=1}^{L} \alpha_{l, i}^{2} .
$$

By substituting equation (20) into equation (19) we obtain the variance expression of $T I_{1}$

$$
V\left[T I_{1}\right]=P^{2} R E\left[x_{k-\tau_{l}, i}^{2}\right] \frac{N_{0}}{2} \sum_{l=1}^{L} \alpha_{l, i}^{2} .
$$

Similarly, the variance of $T I_{2}$ can be expressed as

$$
V\left[T I_{2}\right]=P R \frac{N_{0}^{2}}{4}+P R \frac{N_{0}}{2} \sum_{l=1}^{L} \alpha_{l, i}^{2} E\left[x_{k-\tau_{l}, i}^{2}\right] .
$$

The add-up of the variance components above results in the variance of the decision variable, which becomes

$$
\begin{aligned}
& V\left[D_{i}\right]= \\
& P^{2} R E\left[x_{k-\tau_{l}, i}^{2}\right]\left(\frac{N_{0}}{2} \sum_{l=1}^{L} \alpha_{l, i}^{2}+\sum_{l=1}^{L} \sum_{\substack{l,=1 \\
l, \neq \neq l}}^{L} \alpha_{l, i}^{2} \alpha_{l, i}^{2} E\left[x_{k-\tau_{l}, i}^{2}\right]\right) \\
& +P R \frac{N_{0}^{2}}{4}+P R \frac{N_{0}}{2} \sum_{l=1}^{L} \alpha_{l, i}^{2} E\left[x_{k-\tau_{l}, i}^{2}\right] .
\end{aligned}
$$

Since bit energies (or chaotic chips) are deterministic variables, by virtue of central limit theorem the decision variable at the output of the correlator follows a Gaussian distribution. Therefore, the bit error probability is represented as

$$
\begin{aligned}
\mathrm{BER} & =\frac{1}{2} \operatorname{Pr}\left(D_{i}<0 \mid b_{i}=+1\right) \\
& +\frac{1}{2} \operatorname{Pr}\left(D_{i}>0 \mid b_{i}=-1\right),
\end{aligned}
$$

which takes its final form as

$$
\mathrm{BER}=\frac{1}{2} \operatorname{erfc}\left(\left[\frac{2 \operatorname{Var}\left[D_{i}\right]}{\mathrm{E}\left[D_{i}\right]^{2}}\right]^{-\frac{1}{2}}\right),
$$

where $\operatorname{erfc}(x)$ is the well-known complementary error function defined as $\operatorname{erfc}(x) \equiv \frac{2}{\sqrt{\pi}} \int_{x}^{\infty} e^{-\mu^{2}} d \mu$.

For high reference length $R$, the transmitted bit energy $E_{b}$ can be considered constant. Therefore, for low reference length the energy variation must be taken into account and derived, as done in [26]. In our paper, the bit energy may be considered as constant only if a high value of $R$ is considered. By virtue of equations (18) and (23) above, the BER for the SR-DCSK scheme can be determined and stated as

$$
\mathrm{BER}=\frac{1}{2} \operatorname{erfc}\left(\left[\frac{(\mathrm{R}+\beta) \mathrm{N}_{0}}{\mathrm{R} \sum_{1=1}^{\mathrm{L}} \alpha_{1, \mathrm{i}}^{2} \mathrm{E}_{\mathrm{b}}}\left(\frac{\mathrm{P}+1}{\mathrm{P}}\right)+\frac{(\mathrm{R}+\beta)^{2} \mathrm{~N}_{0}^{2}}{2 \mathrm{RP}\left(\sum_{\mathrm{l}=1}^{\mathrm{L}} \alpha_{1, \mathrm{i}}^{2}\right)^{2} \mathrm{E}_{\mathrm{b}}^{2}}\right]^{-\frac{1}{2}}\right) .
$$

Equation (26) gives the theoretical BER benchmark of SRDCSK system over multipath fading channels. Based on this, it is essential to note that the channel keeps changing at every transmission instant, so the average lower bound on the BER expression becomes

$$
\begin{aligned}
& \overline{\mathrm{BER}}= \\
& \frac{1}{2} \int_{0}^{\infty} \operatorname{erfc}\left(\left[\frac{(\mathrm{R}+\beta)}{\mathrm{R} \gamma}\left(\frac{\mathrm{P}+1}{\mathrm{P}}\right)+\frac{(\mathrm{R}+\beta)^{2}}{2 \mathrm{RP} \gamma^{2}}\right]^{-\frac{1}{2}}\right) f(\gamma) d \gamma,
\end{aligned}
$$

where $\gamma=\sum_{l=1}^{L} \alpha_{l}^{2} E_{b} / N_{0}$.

For a high number of samples $R$, the bit energy $E_{b}$ can be assumed constant [27]. In this case, and for $L$ independent 
and identically distributed (i.i.d) Rayleigh-fading channels, the PDF of the instantaneous $\gamma$ can be written as [28]

$$
f(\gamma)=\frac{\gamma^{L-1}}{(L-1) ! \bar{\gamma}_{c}^{L}} \exp \left(-\frac{\gamma}{\bar{\gamma}_{c}}\right) \equiv f\left(\gamma, \bar{\gamma}_{c}, L\right),
$$

where $\bar{\gamma}_{c}$ is the average SNR per channel defined as

$$
\bar{\gamma}_{c}=\frac{E_{b}}{N_{0}} E\left(\alpha_{j}^{2}\right)=\frac{E_{b}}{N_{0}} E\left(\alpha_{l}^{2}\right), \quad j \neq l .
$$

For dissimilar channels, the PDF of $\gamma$ can be written as [28]

$$
\begin{aligned}
& f(\gamma)=\sum_{l=1}^{L} \frac{\rho_{l}}{\bar{\gamma}_{l}} \exp \left(-\frac{\gamma}{\bar{\gamma}_{l}}\right) \\
& =\sum_{l=1}^{L} \rho_{l} f\left(\gamma, \bar{\gamma}_{l}, 1\right),
\end{aligned}
$$

where

$$
\rho_{l}=\prod_{j=1, j \neq l}^{l} \frac{\bar{\gamma}_{l}}{\bar{\gamma}_{l}-\bar{\gamma}_{j}}
$$

in which $\bar{\gamma}_{l}$ is the average value of $\gamma_{l}=\alpha_{l}^{2} E_{b} / N_{0}$, which is the instantaneous SNR on the $l^{\text {th }}$ path.

Since an analytical solution the integral provided in equation (27) is not easy to find, we will use numerical integration as an alternative solution to compute the average BER. The numerical integration of equation (27) is performed as

$$
B E R \approx \sum_{c=1}^{C} \frac{1}{2} \operatorname{erfc}\left(\left[\frac{(\mathrm{R}+\beta)}{\mathrm{R} \gamma_{c}}\left(\frac{\mathrm{P}+1}{\mathrm{P}}\right)+\frac{(\mathrm{R}+\beta)^{2}}{2 \mathrm{RP} \gamma_{c}^{2}}\right]^{-\frac{1}{2}}\right) f\left(\gamma_{c}\right)
$$

where $C$ is the number of histogram classes and $f\left(\gamma_{c}\right)$ is the probability of having the energy in intervals centred at $\gamma_{c}$. In this work, we have set the number of classes to $C=100$ with a unit integration step size.

Furthermore, equation (26) can be extended to AWGN channel case by substituting $\alpha_{1}=1$ and equating the other path gains to zero. Accordingly, the BER expression in AWGN channels simplifies to

$\mathrm{BER}=\frac{1}{2} \operatorname{erfc}\left(\left[\frac{(R+\beta) N_{0}}{R E_{b}}\left(\frac{P+1}{P}\right)+\frac{(R+\beta)^{2} N_{0}^{2}}{2 R P E_{b}^{2}}\right]^{-\frac{1}{2}}\right)$

\section{ReFERENCE LENGTH ANALYSiS}

In this part of the paper we will analyse the length of the reference $R$ then we will derive the optimal value that minimizes the BER performance of the system. By having a glance at equation (33) we deduce a dependence relationship between the reference length $R$ and the BER performance for a given $\beta$. As a matter of fact, many earlier studies investigated the relationship between the sample length $\beta$ and the performance [7], [29]. In this paper, we optimize the performance of the SR-DCSK system with respect to the reference length $R$ for any value of $\beta$, regardless of $\beta$ being optimized at an earlier stage or not.

In order to understand this relationship we define a function $\Phi(R)$ from equation (33) as

$$
\Phi(R)=\frac{(R+\beta) N_{0}}{R E_{b}}\left(\frac{P+1}{P}\right)+\frac{(R+\beta)^{2} N_{0}^{2}}{2 R P E_{b}^{2}} .
$$

By replacing $P=\frac{\beta}{R}$ and $N_{0}=\frac{E_{b}}{\frac{E_{b}}{N_{0}}}$, the function $\Phi(R)$ shortens to

$$
\Phi(R)=\frac{(R+\beta)^{2}}{R \beta \frac{E_{b}}{N_{0}}}+\frac{(R+\beta)^{2}}{2 \beta\left(\frac{E_{b}}{N_{0}}\right)^{2}} .
$$

Note that $\frac{E_{b}}{N_{0}}$ represents the float value in our equations that reduce equation (35) to the form

$$
\Phi(R)=C_{1} \frac{(R+\beta)^{2}}{R}+C_{2}(R+\beta)^{2},
$$

where $C_{1}=\left[\beta \frac{E_{b}}{N_{0}}\right]^{-1}$ and $C_{2}=\left[2 \beta\left(\frac{E_{b}}{N_{0}}\right)^{2}\right]^{-1}$ are two constants in this function.

Minimizing the BER performance of equation (33) relies on minimizing the function $\Phi(R)$ described by equation (36). The minimization is done by equating the first derivative of $\Phi(R)$ to zero. After straightforward operations, the first derivative of $\Phi(R)$ is

$$
\frac{d \Phi(R)}{d R}=(R+\beta)\left(\frac{2 C_{2} R^{2}+C_{1} R-C_{1} \beta}{R^{2}}\right) .
$$

For $R \neq 0, C_{1} \neq 0, C_{2} \neq 0$ and $(R+\beta) \neq 0$ the solutions for equation (37) that minimizes $\Phi(R)$ are found by solving

$$
2 C_{2} R^{2}+C_{1} R-C_{1} \beta=0
$$

This results in the following minimizing solutions

$$
R_{\text {opt }}=\frac{C_{1} \pm \sqrt{\left(8 \beta C_{1} C_{2}+C_{1}^{2}\right)}}{4 C_{2}} .
$$

where $R_{o p t}$ is the optimal value for a given $\frac{E_{b}}{N_{0}}$ minimizing the function $\Phi(R)$. Note that since $C_{1}$ and $C_{2}$ are directly determined by $E_{b} / N_{0}$, then $R_{o p t}$ is in fact a function of $E_{b} / N_{0}$. Since in general $E_{b} / N_{0}$ can assume any positive real value, i.e. $E_{b} / N_{0} \in \mathbb{R}^{+}$, according to equation (39) the calculated $R_{\text {opt }}$ may assume any positive real value too. This becomes clear when we replace $C_{1}$ and $C_{2}$ and consider only the positive value of $R_{o p t}$, which can be written in terms of $E_{b} / N_{0}$ as

$$
R_{o p t}=\frac{E_{b}}{2 N_{0}}\left(1+\sqrt{1+\frac{4 \beta N_{0}}{E_{b}}}\right) .
$$

Fig. 3 plots the function $\Phi(R)$ for $E_{b} / N_{0}=5,10$ and $15 \mathrm{~dB}$ when $\beta=100$. For these parameters, equation (40) gives the following optimal values $R_{o p t}=19.4,37.05$, and 74.2261 for $E_{b} / N_{0}=15,10$ and $5 \mathrm{~dB}$ respectively. It must be remarked that since $P$ is an integer, $\beta$ will be an integer 
multiple of $R$ and this is a condition that must be satisfied, in the sense that $\beta$ must always be dividable by $R$. Furthermore, in cases where the optimal value mismatches the values of $R$, the closest value to $R_{o p t}$ either from left or from right is considered. The left or right choice depends on the data rate requirements and the wireless channel. In fact, since this system will operate in a multipath fading channel, the length of the reference sequence must be higher than the maximum delay spread of the channel in order to avoid or reduce ISI, i.e. $R>\tau_{L}$.

The arrows in the figure show the limits where the values of $R$ yield better BER performance than DCSK system (i.e. $R=\beta=100)$. The results obtained and presented in this section demonstrate for the first time that the optimal length of the reference signal is not necessarily equal to the length of the data carrier, as widely adopted in DCSK systems. In fact, unlike the conventional DCSK, there exist many other values of $R$, which result in lower values of $\Phi(R)$ for different noise variance level.

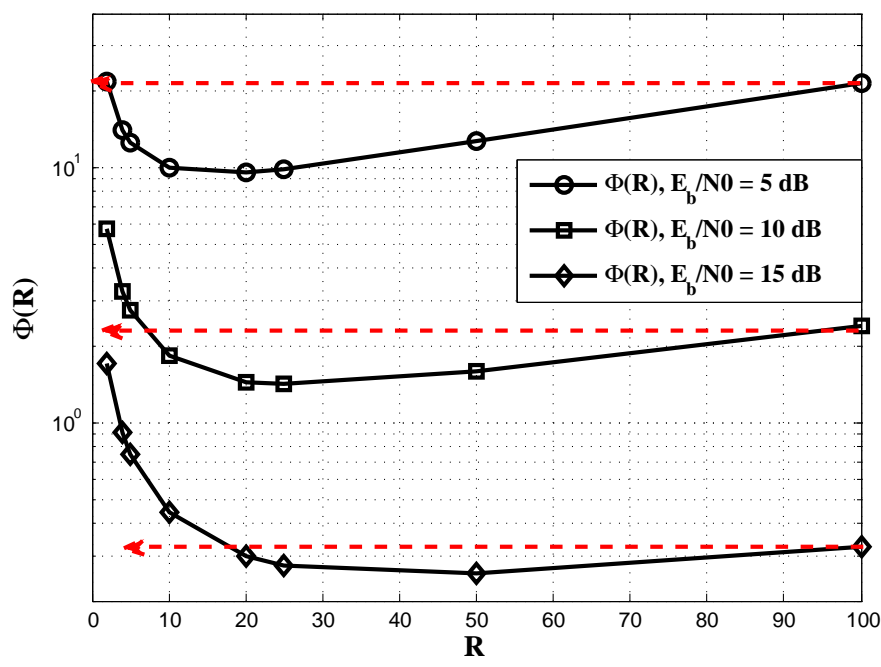

Fig. 3: $\Phi(R)$ function versus $R$ for $E_{b} / N_{0}=5,10$ and $15 \mathrm{~dB}$ and $\beta=100$.

\section{Discussions And Simulation Results}

In order to validate the performance of the SR-DCSK scheme and compare it to existing chaos-based and conventional non-coherent schemes, the computed BER expressions are verified and well justified by simulation results under AWGN and multipath fading channels. Moreover, since the condition $0<\tau_{L}<<R$ is respected, ISI becomes negligible and the BER performance will not be influenced whether $\tau_{L}$ is chosen statically or is dynamically varying within the above range.

The effect of the reference signal length $R$ on the performance of SR-DCSK in AWGN channels is studied and the outcome is depicted in Fig. 4. Simulated and analytical bit error rate performance are plotted for different values of the reference signal length $R$ with a fixed $E_{b} / N_{0}$ and length of data carrier $\beta=100$. To validate our analysis regarding the length of the reference signal, the analytical BER is evaluated using the $\Phi(R)$ function by selecting BER = $0.5 \operatorname{erfc}\left(\Phi(\mathrm{R})^{-\frac{1}{2}}\right)$.

We can clearly see a perfect match between simulation and analytic expressions. In addition, simulation results are in total agreement with the results portrayed in Fig. 3. This indicates that the reference length values close the optimal value for either $E_{b} / N_{0}=10$ or $E_{b} / N_{0}=17$ do indeed minimize the bit error rate. Moreover, we also observe that $E_{b} / N_{0}=10$, $R=20,25$, and 50 yield close (near) overlapping and indistinguishable BER performances. In this case, we choose the reference length that minimizes the ISI by observing the condition $\tau_{L}<R$. Making such a choice allows the system to deliver good performances. A noticeable observation here is that there exists a trade-off regarding the multipath channel between performance, data rate and energy efficiency.

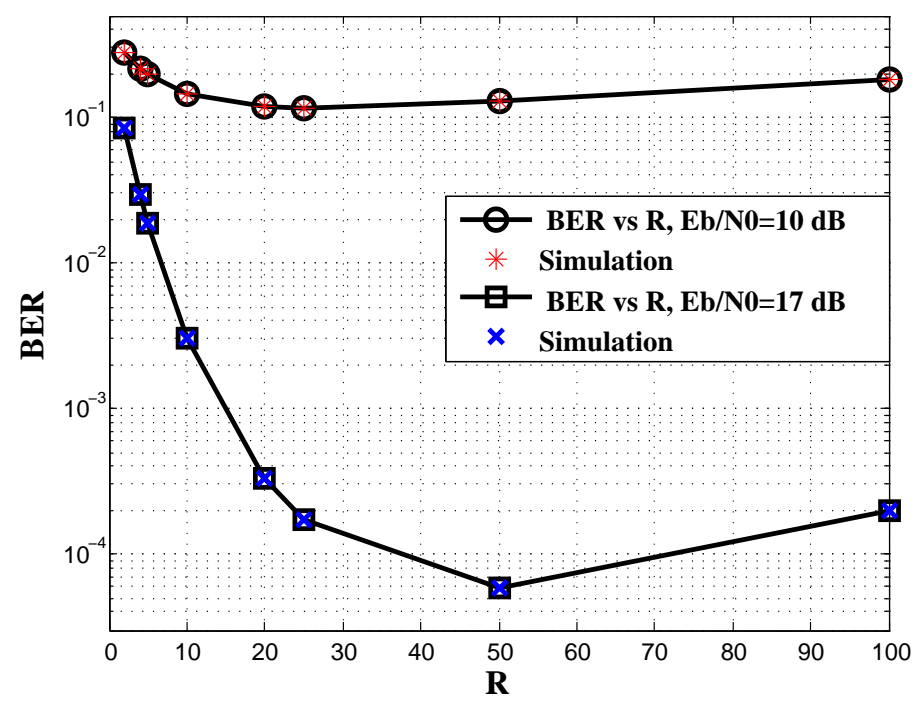

Fig. 4: Simulation of the BER for a signal carrier length of $\beta=100$ versus different values of $R$ over AWGN channel.

Performance results of SR-DCSK in AWGN channels for $\beta=100$ and for various values of the reference signal length $R$ is shown in Fig. 5. As can be perceived in Fig. 5, simulation results perfectly validate the analytical BER expression given by equation (33). It is clearly observed that the optimized value of $R=50$ results in a superior performance. Moreover, SR-DCSK system performance at $\beta=R=100$ represents in fact the performance of conventional DCSK. In addition, SR-DCSK outperforms the DCSK system when $R=20$ for $E_{b} / N_{0}$ values $\leq 15 \mathrm{~dB}$.

Eventually, by choosing $R=20$ or $R=50$, SR-DCSK delivers a better performance than DCSK and according to equations (5), (7) and Fig. 2 in such circumstances, the SRDCSK system boosts its data rate by $65 \%$ and $33 \%$ and saves $40 \%$ and $25 \%$ from the bit energy, respectively. In order to show and elaborate that the proposed approach may very well be applied to a majority of transmit reference modulation classes, a performance comparison of SR-DCSK, QCSK and MC-DCSK has been carried out. Fig. 6 shows the performance of QCSK and MC-DCSK systems with and without the shortened reference approach. By inspecting this figure, we 


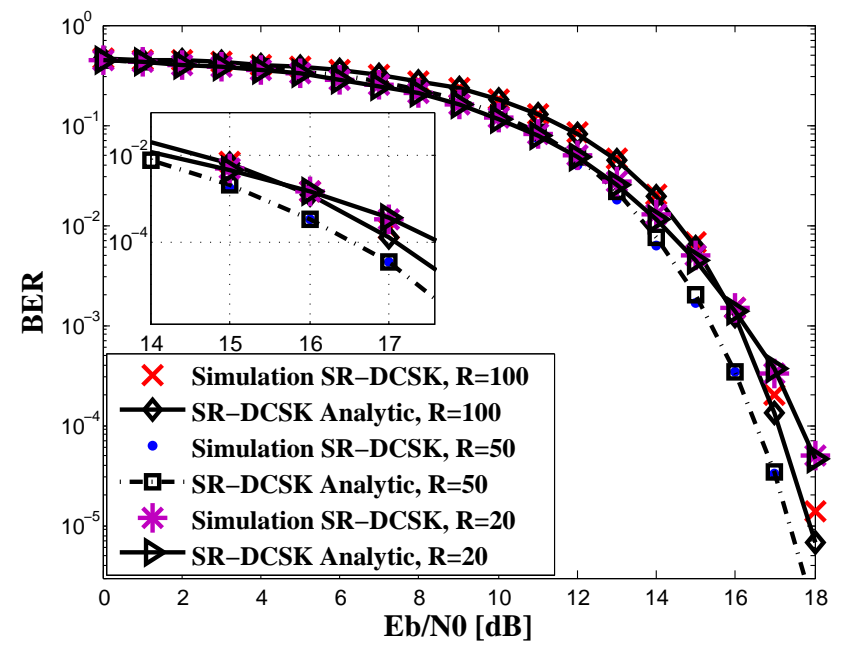

Fig. 5: Simulation and analytical BER performance of SR-DCSK and DCSK in AWGN channels for $\beta=100$ and various $R$.

can see that the performance is more improved for QCSK than MC-DCSK systems when $R=50$. This is due to the fact that in QCSK systems, performance enhancements can in interpreted and evaluated as done in DCSK systems. Since the reference is shared with $M-1$ bits [7] in MC-DCSK systems, shortening the reference signal length makes the effective bit energy equal to $E_{b}=\frac{((M-1) \beta-R) E\left[x_{k}^{2}\right]}{(M-1)}$ and the values of $R$ will slightly influence the energy which leads to the slow improvement of the BER performance. Therefore, applying the SR approach will increase the data rate and enhance the energy efficiency of the system. The obtained BER performance under

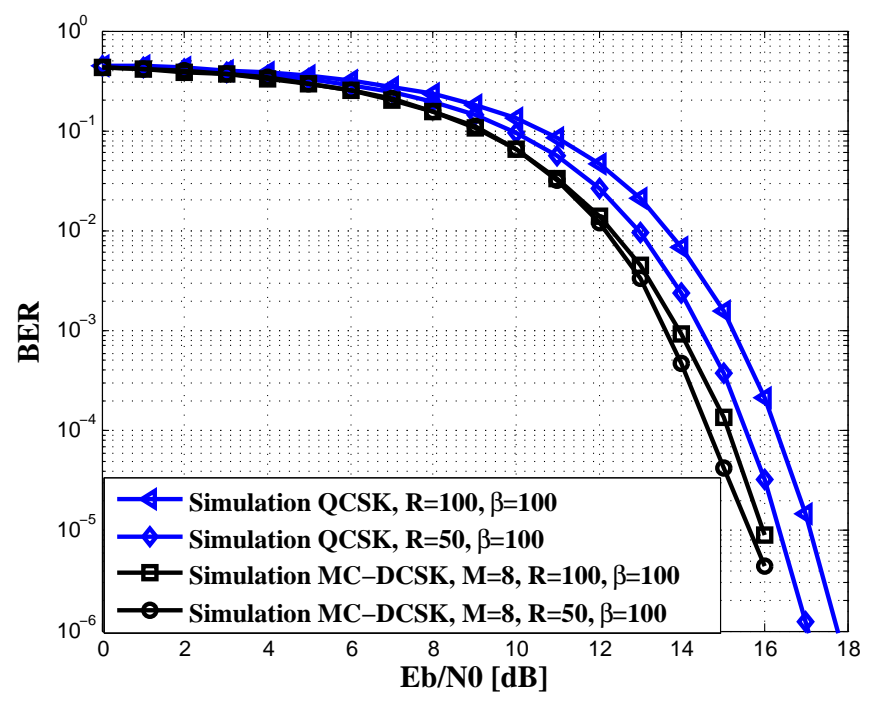

Fig. 6: BER performance of QCSK and MC-DCSK for $\beta=100$ versus $E_{b} / N_{0}$ in AWGN channels (simulation).

multipath Rayleigh fading for a spreading factor $\beta=100$, $L=4$ and for $R=25,50$ and 100 is shown in Fig. 7. The average power gain for the first through the fourth paths are $E\left[\alpha_{1}^{2}\right]=0.5, E\left[\alpha_{2}^{2}\right]=0.3, E\left[\alpha_{3}^{2}\right]=0.15$ and $E\left[\alpha_{4}^{2}\right]=0.05$, in accordance with the set of delays $\tau_{1}=0, \tau_{2}=T_{c}, \tau_{3}=2 T_{c}$ and $\tau_{4}=5 T_{c}$ that exists between the paths, respectively. Simulation results affirm the accuracy of our BER expression given by equation (27) for $R=100$. Since the analytical results provide a lower bound on the BER performance where ISI is totally ignored, we have investigated the limit to which this assumption about neglecting ISI is valid via decreasing $\mathrm{R}$. Therefore, the gap between simulation and analytical BER performance curves is expected to widen as $\mathrm{R}$ is reduced. Naturally, as R is decreased from 50 to 25 for example, ISI becomes more influential and the BER performance degrades as witnessed in Fig. 7. Note that the case of $R=\beta=100$ corresponds to the conventional DCSK in Fig. 7. In addition, these results confirm two additional important properties to be added to the advantages of SR-DCSK system. The first one is the resistance to multipath propagation while being simple to implement. The second one is that this system can perform without the need of CSI at the receiver to recover the data. Finally, the choice of $R=25$ or $R=50$ are to be made according to the channel characteristics, i.e. if less ISI interference is desired or if higher data rates and more energy savings are sought. Nonetheless, the system will still continue to have the essential proprieties of chaos-based non-coherent detection with higher data rates and more efficient energy consumption in both situations. Finally, to demonstrate the

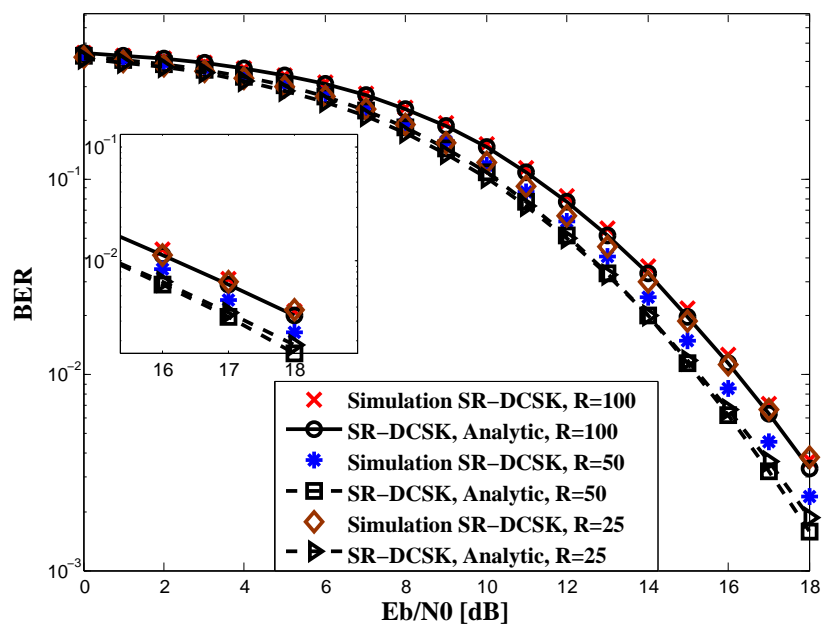

Fig. 7: BER performance of SR-DCSK and DCSK systems in multipath Rayleigh fading channels for $\beta=100, L=4$ and $R=25,50,100$.

effects of the multipath channel on the system performance, we have simulated the SR-DCSK system for different number of paths $L$ with equiproportional gains that satisfy $\sum_{l=1}^{L} \alpha_{l, i}^{2}=1$. The path delays are chosen randomly while observing the condition $\tau_{l}<<R$ in order to keep ISI negligible. The results of this specific simulation which are shown in Fig. 8 indicate that the SR-DCSK system takes advantage of the space diversity offered by the multipath environment to enhance the BER performance when the number of paths increases. 


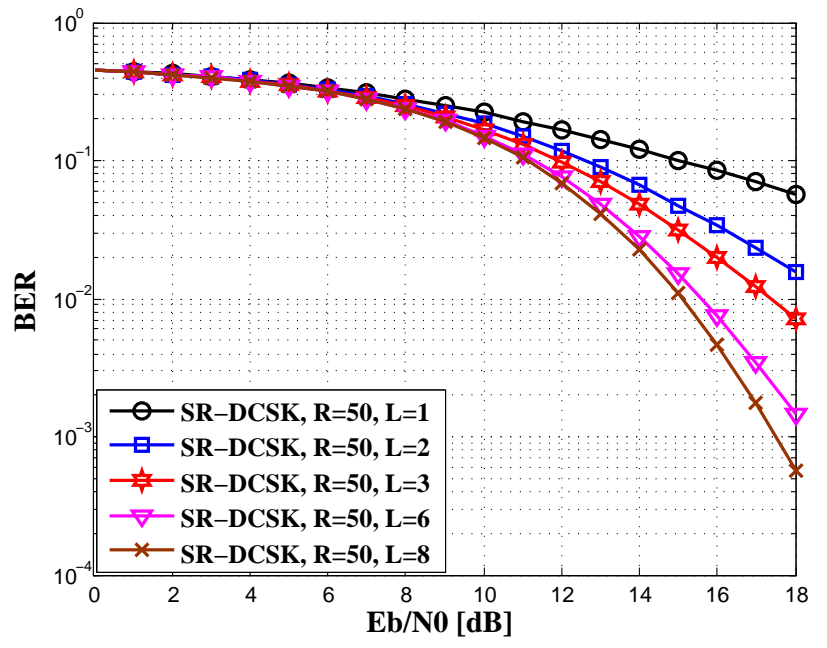

Fig. 8: BER performance of SR-DCSK over multipath Rayleigh fading channels for $\beta=100, R=50, L=1,2,3,6,8$ and equiproportional path gains.

\section{CONCLUSIONS}

A short reference SR-DCSK system is proposed in this paper. The aim of this system is to improve data rate and save bit energy while preserving the advantages of the conventional DCSK and being easy to implement. At the transmitter, $R$ samples of the reference signal are first generated and then replicated $P$ times to build the data carrier signal. This operation aims at making the reference signal shorter than the data carrier signal to reduce the frame duration, leading to data rate escalation and bit energy saving. At the receiver, the reference signal is partially correlated $P$ times with the data carrier signal and the resultant output is compared to a threshold in order to recover the transmitted data. Having presented the system as such, data rate enhancements and energy saving percentages are derived and the performance of the SR-DCSK is analytically studied in order to come up with a general bit error rate expression under multipath fading channels. The analytical BER expression is then simplified to suit the AWGN channel scenario. Moreover, the optimal length of the reference signal is computed and analysed. The results show for the first time that the optimal value of $R$ is not equal to the length of the data carrier signal as widely speculated in the existent literature for transmit reference non-coherent chaos-based communication systems. Computer simulations are carried out to enrich our study and confirm the derived analytical results. Indeed, the results obtained in this work show that the proposed system outperforms the conventional DCSK system in both AWGN and multipath fading channels. Moreover, the SR-DCSK system increases the data rate by $65 \%$ and $33 \%$ and saves by $40 \%$ and $25 \%$ the bit energy if the length $R$ is equal to half or quarter of the data carrier signal length $\beta$, respectively. In conclusion, taking BER performance, data rate, energy efficiency and complexity issues into consideration, the overall performance of our proposed system is promising and may pave the way for the establishment of new generations of non-coherent transmit reference chaos-based communication systems.

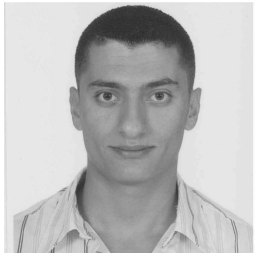

Georges Kaddoum (M'11) received the Bachelor's degree in electrical engineering from the École $\mathrm{Na}$ tionale Supérieure de Techniques Avancées (ENSTA Bretagne), Brest, France, and the M.S. degree in telecommunications and signal processing (circuits, systems, and signal processing) from the Université de Bretagne Occidentale and Telecom Bretagne(ENSTB), Brest, in 2005 and the Ph.D. degree (with honors) in signal processing and telecommunications from the National Institute of Applied Sciences (INSA), University of Toulouse, Toulouse, France, in 2009. Since november 2013, he is an Assistant Professor of electrical engineering with the École de Technologie Supérieure (ETS), University of Quebec, Montréal, QC, Canada. In 2014, he was awarded the ETS Research Chair in physical-layer security for wireless networks. Since 2010, he has been a Scientific Consultant in the field of space and wireless telecommunications for several companies (Intelcan Techno-Systems, MDA Corporation, and Radio-IP companies). He has published over 70 journal and conference papers and has two pending patents. His recent research activities cover wireless communication systems, chaotic modulations, secure transmissions, and space communications and navigation. Dr. Kaddoum received the Best Paper Award at the 2014 IEEE International Conference on Wireless and Mobile Computing, Networking, and Communications, with three coauthors, and the 2015 IEEE Transactions on Communications Top Reviewer Award.

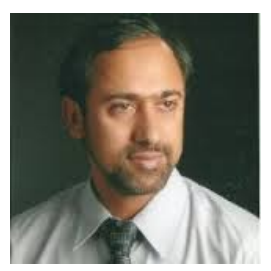

Ebrahim Soujeri (SM'03) received the B.Sc., M.Sc. and $\mathrm{Ph} . \mathrm{D}$. degrees in Electrical and Electronics Engineering from Eastern Mediterranean University, Cyprus, in 1995, 1997 and 2003, respectively. He has worked at the department of E.E.Eng. of EMU during the period 1996 to 2002 as a research assistant while pursuing his M.Sc. and Ph.D. degrees. In 2003, he joined the faculty of Engineering at the European University of Lefke in Cyprus. In 2007, he was a visiting Assist. Prof. at Sultan Qaboos University in Sultanate of Oman. He will be pursuing his second Ph.D. from September 2014 at École de Technologie Supérieure, Montréal, QC, Canada. His research activities cover wireless communication systems, spread spectrum and index modulation techniques.

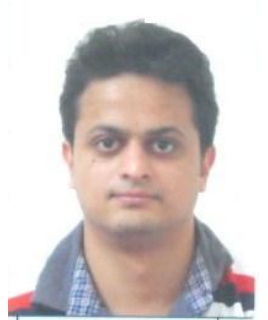

Yogesh Nijsure (M'12) received the B.E. degree (Distinction) in Electronics Engineering from University of Mumbai, India, in June 2006 and received his M.Sc. degree (Distinction) in Wireless Communication Systems Engineering from the University of Greenwich, U.K. in September 2008. He received his Ph.D. degree from University of Newcastle upon Tyne in U.K. in October 2012. From March 2010 to September 2010 he undertook his research internship at the Institute for Infocomm Research (I2R), Singapore, as a research engineer. From November 2011 to November 2012 he worked as a research associate at Nanyang Technological University, Singapore. From December 2012 to April 2014 he worked at Rockwell Collins India Pvt. Ltd. as a Technical Specialist Systems within the aerospace and defense research domain. Since May 2014, he is working as a post-doctoral research fellow at École de Technologie Supérieure (ETS), University of Quebec, Montreal, QC, Canada. He is a member of IEEE and has authored several Top-Tier IEEE conferences and IEEE Transaction journal publications. Till date he has submitted two patent applications in U.S.A and India respectively. His research interests include cognitive radar network design, Bayesian non-parametric methods for wireless sensor network applications, UWB radar systems, cognitive radio networks, wireless communication theory, information theory and radar signal processing, electronic warfare and software defined radio systems. 


\section{REFERENCES}

[1] F. C. M. Lau and C. K. Tse, Chaos-Based Digital communication systems. Springer-Verlag, 2003.

[2] M. P. Kennedy, G. Kolumbán, G. Kis, and Z. Jákó, "Performance evaluation of FM-DCSK modulation in multipath environments," IEEE Trans. Circuits Syst. I, Fundam. Theory Appl., 2000.

[3] A. P. Kurian, S. Puthusserypady, and S. M. Htut, "Performance enhacment of DS-CDMA system using chaotic complex spreading sequence," IEEE Trans. Wireless Commun., vol. 4, no. 3, pp. 984-989, May 2005.

[4] R. Vali, S. Berber, and S. K. Nguang, "Accurate derivation of chaosbased acquisition performance in a fading channel," IEEE Trans. Wireless Commun., vol. 11, no. 2, pp. 722 -731, Feb. 2012.

[5] R. Vali, S. Berber, and S.-K. Nguang, "Analysis of chaos-based code tracking using chaotic correlation statistics," IEEE Trans. Circuits and Syst. I: Reg. Papers, vol. 59, no. 4, pp. 796-805, 2012.

[6] S. Berber and S. Feng, "Chaos-based physical layer design for WSN applications," in Recent Advances in Telecommunications and Circuit Design (CIRCOM 2013), vol. 2, 2013, pp. 157-162.

[7] G. Kaddoum, F. Richardson, and F. Gagnon, "Design and analysis of a multi-carrier differential chaos shift keying communication system," IEEE Trans. on Commun., vol. 61, no. 8, pp. 3281-3291, 2013.

[8] V. Lynnyk and S. Celikovsky, "On the anti-synchronization detection for the generalized Lorenz system and its application to secure encryption," Kybernetika, vol. 46, pp. 1-18, 2010.

[9] J. Yu and Y.-D. Yao, "Detection performance of chaotic spreading LPI waveforms," IEEE Trans. Wireless Commun., vol. 4, no. 2, pp. 390 396, March 2005

[10] Y. Xia, C. K. Tse, and F. C. M. Lau, "Performance of differential chaosshift-keying digital communication systems over a multipath fading channel with delay spread," IEEE Trans. Circuits and Syst. II: Exp. Briefs, vol. 51, pp. 680-684, 2004.

[11] B. Jovic, "Chaos-based BPSK communication system," Electronics Letters, vol. 51, no. 8, pp. 630-632, 2015.

[12] Y. Fang, L. Wang, and G. Chen, "Performance of a multiple-access DCSK-CC system over nakagami-m fading channels," in Proc. 2013 IEEE International Symposium on Circuits and Systems (ISCAS), May 2013, pp. 277-280.

[13] P. Chen, L. Wang, and F. Lau, "One analog STBC-DCSK transmission scheme not requiring channel state information," IEEE Trans. Circuits and Syst. I: Reg. Papers, vol. 60, no. 4, pp. 1027-1037, 2013.

[14] J. Schweizer and T. Schimming, "Symbolic dynamics for processing chaotic signals- I: noise reduction of chaotic sequence," IEEE Trans. Circuits Syst. I, Fundam. Theory Appl., vol. 48, pp. 1269-1282, 2001.

[15] G. Cimatti, R. Rovatti, and G. Setti, "Chaos-Based Spreading in DSUWB Sensor Networks Increases Available Bit Rate," IEEE Trans. on Circuits and Syst. I: Reg. Papers, vol. 54, no. 6, pp. 1327-1339, June 2007.
[16] W. Xu, L. Wang, and G. Chen, "Performance of DCSK cooperative communication systems over multipath fading channels," IEEE Trans. on Circuits and Systems I: Reg. Papers, vol. 58, no. 1, pp. 196-204, Jan 2011.

[17] G. Kaddoum and E. Soujeri, "NR-DCSK: A noise reduction differential chaos shift keying system," IEEE Trans. on Circuits and Syst. II: Exp. Briefs, vol. PP, no. 99, pp. 1-5, 2015.

[18] Z. Zhibo, Z. Tong, and W. Jinxiang, "Performance of Multiple-Access DCSK Communication over a Multipath Fading Channel with Delay Spread," Circuits, Systems and Signal Processing, vol. 27, pp. 507-518, 2008.

[19] S. Wang and X. Wang, "M-DCSK based chaotic communications in MIMO multipath channels with no channel state information," IEEE Trans. on Circuits and Syst. II: Exp. Briefs, vol. 57, no. 12, pp. 10011005, Dec. 2010

[20] Z. Galias and G. M. Maggio, "Quadrature chaos shift keying: Theory and performance analysis," IEEE Trans. Circuits Syst. I, Fundam. Theory Appl., 2001.

[21] L. Wang, G. Cai, and G. Chen, "Design and performance analysis of a new multiresolution M-ary differential chaos shift keying communication system," IEEE Trans. on Wireless Commun., vol. 14, no. 9, pp. 5197-5208, Sept 2015.

[22] H. Yang and G.-P. Jiang, "High-efficiency differential-chaos-shift-keying scheme for chaos-based noncoherent communication," IEEE Trans Circuits and Syst. II: Exp. Briefs, vol. 59, no. 5, pp. 312 -316, May 2012.

[23] G. Kaddoum and F. Shokraneh, "Analog network coding for multiuser multi-carrier differential chaos shift keying communication system,"

IEEE Trans. on Wireless Commun., vol. 14, no. 3, pp. 1492-1505, March 2015.

[24] G. Kaddoum, "Design and performance analysis of a multi-user OFDM based differential chaos shift keying communication system," IEEE Trans. on Commun., vol. PP, no. 99, pp. 1-12, 2015.

[25] G. Kaddoum, E. Soujeri, C. Arcila, and K. Eshteiwi, "I-DCSK: An improved non-coherent communication system architecture," IEEE Trans. on Circuits and Syst. II: Exp. Briefs, vol. PP, no. 99, pp. 1-5, 2015.

[26] G. Kaddoum, P. Chargé, D. Roviras, and D. Fournier-Prunaret, "A methodology for bit error rate prediction in chaos-based communication systems," Birkhäuser, Circuits, Systems and Signal Processing, vol. 28, pp. 925-944, 2009

[27] G. Kaddoum, P. Chargé, and D. Roviras, "A generalized methodology for bit-error-rate prediction in correlation-based communication schemes using chaos," IEEE Commun. Letters., vol. 13, no. 8, pp. 567-569, 2009.

[28] J. G. Proakis, Digital communications. McGraw-Hill, 2001.

[29] M. Sushchik, L. S. Tsimring, and A. R. Volkovskii, "Performance analysis of correlation-based communication schemes utilizing chaos," IEEE Trans. Circuits Syst. I, Fundam. Theory Appl., vol. 47, no. 12, pp. $1684-1691,2000$ 